\title{
Essential spectrum of a periodic waveguide with non-periodic perturbation
}

\section{Nazarov, Sergei A.}

2018-07-15

Nazarov , S A \& Taskinen , J 2018 , ' Essential spectrum of a periodic waveguide with non-periodic perturbation ' , Journal of Mathematical Analysis and Applications, vol. 463 , no. 2 , pp. 922-933 . https://doi.org/10.1016/j.jmaa.2018.03.057

http://hdl.handle.net/10138/314498

https://doi.org/10.1016/j.jmaa.2018.03.057

cc_by_nc_nd

acceptedVersion

Downloaded from Helda, University of Helsinki institutional repository.

This is an electronic reprint of the original article.

This reprint may differ from the original in pagination and typographic detail.

Please cite the original version. 


\title{
ESSENTIAL SPECTRUM OF A PERIODIC WAVEGUIDE WITH NON-PERIODIC PERTURBATION
}

\author{
SERGEI A. NAZAROV AND JARI TASKINEN
}

\begin{abstract}
We consider the spectral Dirichlet-Laplacian problem on a domain which is formed from a periodic waveguide $\Pi$ perturbed by non-compact, nonperiodic changes of geometry. We show that the domain perturbation causes an addition to the essential spectrum, which consists of isolated points belonging to the discrete spectrum of a model problem. This model problem is posed on a domain, which is just a compact perturbation of $\Pi$. We discuss the position of the new spectral components in relation to the essential spectrum of the problem in $\Pi$.
\end{abstract}

\section{INTRODUCTION AND FORMULATION OF THE PROBLEMS.}

We consider the effect of non-compact domain perturbations to the essential spectrum of the Dirichlet-Laplacian. To describe our result, we assume that a 1-periodic quasicylinder $\Pi$ in the Euclidean space $\mathbb{R}^{d}, d \geq 2$, as well as a sequence $\left(\ell_{j}\right)_{j=1}^{\infty}$ of natural numbers tending to $+\infty$ be given. The domain $\Pi$ is perturbed by an infinite family of identical cells, such that the neighboring ones are situated at the distance $\ell_{j}$ of each other; see Fig. 1.1, 4.1. Our main result, Theorem 3.1, states that the essential spectrum, denoted later by $\sigma_{\text {ess }}\left(A_{\bullet}\right)$, of the perturbed problem is the union of two components: the first one consists of the essential spectrum of the unperturbed problem and the second one of the discrete spectrum of a model problem, which is a spectral problem on the domain $\Pi$ perturbed only by a single cell.

One of the conclusions is that the result does not depend on the growth rate of the sequence $\left(\ell_{j}\right)_{j=1}^{\infty}$; in particular, the domain perturbation is non-periodic and can be made as "sparse" as one wishes. However, it is essential for the result and its proof that the sequence $\left(\ell_{j}\right)_{j=1}^{\infty}$ is unbounded. The result should be compared with the papers [6], [1], [20], which contain analysis of the essential spectra of elliptic boundary problems in doubly periodic planar domains with domain perturbation consisting of semi-infinite open waveguides. These perturbations differ from those in the present paper, as they still have periodic structure with the same periodicity dimension as the intact domain and thus are rather analogous with the case of a constant sequence $\left(\ell_{j}\right)_{j=1}^{\infty}$. In [6] it shown that the essential spectrum consist of two components, one coming from the corresponding problem in the unperturbed domain and another one related to a family of model problems on the periodic domain perturbation. In both [6] and [20] there are explicit examples how the insertion of open waveguides into the domain increases the essential spectrum.

We proceed to detailed descriptions of the domains and equations to be investigated. The wavequide $\Pi$ is assumed to be a domain in $\mathbb{R}^{d}, d \geq 2$, in particular a

The first named author was partially supported by the grant 15-01-02175 of the Russian Foundation of Basic Research and the grant 308829 of the Academy of Finland. The second named author was partially supported by grants from the Faculty of Science of the University of Helsinki and from the Väisälä Foundation of the Finnish Academy of Science and Letters. 


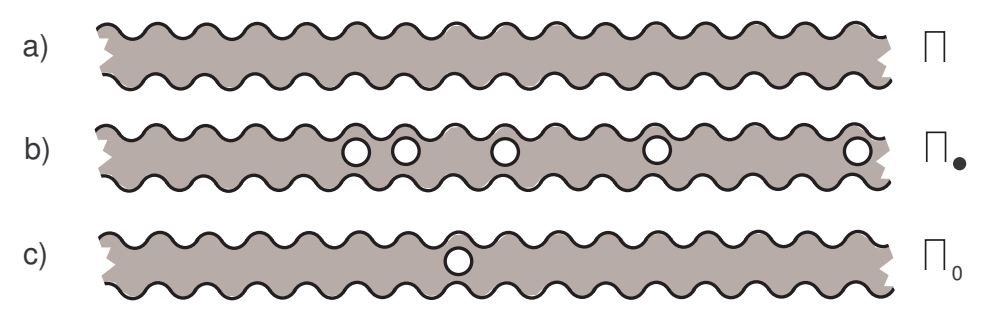

FiguRe 1.1. Intact (a), perturbed (b), and model (c) waveguides.

connected set, such that the boundary $\partial \Pi$ is a smooth $(d-1)$-dimensional surface. Moreover, $\Pi$ is assumed to be a 1-periodic quasicylinder contained inside a circular cylinder of radius $R>0$,

$$
\Pi \subset\left\{x=(y, z) \in \mathbb{R}^{d-1} \times \mathbb{R}:|y|<R, z \in \mathbb{R}\right\} .
$$

By the periodicity we mean that

$$
\Pi=\left\{x=(y, z) \in \mathbb{R}^{d}:(y, z \pm 1) \in \Pi\right\},
$$

and we denote the periodicity cell of $\Pi$ by

$$
\varpi=\{x \in \Pi: z \in(0,1)\} .
$$

and the translations of the cell $\varpi=: \varpi(1)$ by

$$
\varpi(n)=\varpi+(0, \ldots, 0, n-1)=\{x \in \Pi: z \in(n-1, n)\}, \quad n \in \mathbb{N} .
$$

We consider the spectral Dirichlet problem for the Laplace operator $\Delta=\nabla \cdot \nabla$,

$$
-\Delta u(x)=\lambda u(x) \text { for } x \in \Pi, \quad u(x)=0 \text { for } x \in \partial \Pi .
$$

The left-hand side of the variational formulation

$$
(\nabla u, \nabla v)_{\Pi}=\lambda(u, v)_{\Pi} \quad \forall v \in H_{0}^{1}(\Pi)
$$

contains a positive and closed bilinear form in $H_{0}^{1}(\Pi)$, and thus problem (1.4) is associated with a positive self-adjoint operator $A$ with domain $\mathcal{D}(A)=H^{2}(\Pi) \cap$ $H_{0}^{1}(\Pi)$; see [24, Thm. VIII.15], [4, Ch. 10]. Here, $(\cdot, \cdot)_{\Pi}$ is the natural scalar product in the Lebesgue space $L^{2}(\Pi)$, while $H^{2}(\Pi)$ and $H_{0}^{1}(\Pi)$ are standard Sobolev spaces, the latter with the homogeneous Dirichlet condition on $\partial \Pi$. It is known that the essential spectrum $\sigma_{\text {ess }}(A)$ of $A$ has the band-gap structure

$$
\sigma_{\mathrm{ess}}(A)=\bigcup_{k \in \mathbb{N}} \beta(k),
$$

where $\mathbb{N}=\{1,2,3, \ldots\}$ and $\beta(k)$ are compact intervals contained in $\mathbb{R}_{+}=(0, \infty)$; see for example [25], [13]. The spectral bands $\beta(k)$ are described by means of a model spectral problem in the periodicity cell $\varpi$, see (1.9).

Let a bounded, perturbed cell $\varpi_{\bullet} \subset\{x: z \in(0,1)\}$ with $\varpi \bullet \neq \varpi$ be given. The following translations

$$
\begin{aligned}
\varpi_{\bullet}\left(L_{j}\right) & =\left\{x:\left(y, z-L_{j}\right) \in \varpi_{\bullet}\right\}, \text { where } j \in \mathbb{N} \text { and } \\
L_{j} & =\ell_{1}+\ldots+\ell_{j}, \quad \ell_{k} \in \mathbb{N} \backslash\{1\},
\end{aligned}
$$


are used to define the perturbed waveguide $\Pi$. by replacing in (1.1)-(1.3) the cells $\varpi\left(L_{j}\right)$ by $\varpi_{\bullet}\left(L_{j}\right)$ for all $j \in \mathbb{N}$, see Fig. $\left.1.1, \mathrm{~b}\right)$. We assume about the geometry that $\Pi$. becomes a domain with smooth boundary. Then, let us consider the problem

$$
-\Delta u_{\bullet}(x)=\lambda u_{\bullet}(x) \text { for } x \in \Pi_{\bullet}, u_{\bullet}(x)=0 \text { for } x \in \partial \Pi_{\bullet} .
$$

In the same way as above, this problem is associated with a positive self-adjoint operator $A_{\bullet}$ in $L^{2}\left(\Pi_{\bullet}\right)$.

If all the numbers $\ell_{j}$ equal a constant, then $\Pi_{\bullet}$ remains as a periodic quasicylinder so that (1.8) reduces to (1.4) by a rescaling. Instead, we next consider the case $\left\{\ell_{k}\right\}_{k \in \mathbb{N}}$ is a sequence such that $\lim _{j \rightarrow \infty} \ell_{j}=+\infty$. As a consequence, the waveguide $\Pi$. loses the periodicity, and the description of the essential spectrum $\sigma_{\text {ess }}\left(A_{\bullet}\right)$ of the problem (1.8) becomes the main goal of our paper.

The model problem in the cell (1.2) reads as

$$
\begin{aligned}
&-\left(\Delta_{y}+\left(\partial_{z}+i \eta\right)^{2}\right) U(x ; \eta)=\Lambda(\eta) U(x ; \eta), \quad x \in \varpi \\
& U(x ; \eta)=0, \quad x \in \kappa \\
& U(y, 1 ; \eta)=U(y, 0 ; \eta), \partial_{z} U(y, 1 ; \eta)=\partial_{z} U(y, 0 ; \eta), \quad(y, 0) \in \tau,
\end{aligned}
$$

where the Dirichlet condition is kept on the lateral side $\kappa=\{z \in \partial \Pi: z \in(0,1)\}$ of the cell and the periodicity conditions are imposed on the cross-section $\tau=\{z \in$ $\Pi: z=0\}$. For all values of the Floquet parameter $\eta \in[-\pi, \pi]$, the spectrum of the problem (1.9) is discrete and consists of a positive unbounded sequence of eigenvalues listed according to their multiplicities,

$$
0<\Lambda_{1}(\eta) \leq \Lambda_{2}(\eta) \leq \ldots \leq \Lambda_{n}(\eta) \leq \ldots \rightarrow+\infty
$$

The functions $[-\pi, \pi] \ni \eta \mapsto \Lambda_{n}(\eta)$ are continuous and $2 \pi$-periodic. Hence, according to $[25,13]$, the spectral bands

$$
\beta(n):=\left\{\Lambda_{n}(\eta): \eta \in[-\pi, \pi]\right\} \subset \mathbb{R}_{+}
$$

are indeed compact intervals. Moreover, $\lambda_{\dagger}=\Lambda_{1}(0)>0$ is the minimum of $\sigma_{\text {ess }}(A)$ because $\Lambda_{1}(0)<\Lambda_{1}(\eta)$ for $\eta \neq 0$.

The other auxiliary problem, needed later, is the Dirichlet problem

$$
-\Delta w(x)=\lambda w(x) \text { for } x \in \Pi_{0}, w(x)=0 \text { for } x \in \partial \Pi_{0},
$$

in the infinite waveguide $\Pi_{0}$, Fig. 1.1,c), which is obtained from $\Pi,(1.1)-(1.3)$, by only replacing the "central" cell $\varpi \mapsto \varpi_{\bullet}$. Also the problem (1.12) is supplied with a positive self-adjoint operator $A_{0}$ in the space $L^{2}\left(\Pi_{0}\right)$, the essential spectrum $\sigma_{\text {ess }}\left(A_{0}\right)$ of which coincides with (1.6), i.e. $\sigma_{\text {ess }}\left(A_{0}\right)=\cup_{k \in \mathbb{N}} \beta(k)$. However, it may also have discrete spectrum $\sigma_{\mathrm{di}}\left(A_{0}\right)$, which consists of isolated eigenvalues either in the interval $\gamma(0):=\left(0, \lambda_{\dagger}\right)$ below the essential spectrum, or inside a spectral gap $\gamma(n)=\left(\beta^{+}(n), \beta^{-}(n+1)\right) \neq \varnothing$ between disjoint neighboring bands

$$
\beta(n)=\left[\beta^{-}(n), \beta^{+}(n)\right] \text { and } \beta(n+1)=\left[\beta^{-}(n+1), \beta^{+}(n+1)\right] .
$$

Examples of such eigenvalues will be given in Section 4. In any case every eigenfunction $w \in H^{2}\left(\Pi_{0}\right) \cap H_{0}^{1}\left(\Pi_{0}\right)$ of the problem (1.12), corresponding to a spectral parameter $\mu \in \sigma_{\mathrm{di}}\left(A_{0}\right)$ as $\lambda$, has exponential decay at infinity, namely

$$
e^{\beta(\mu)|z|} w \in H_{0}^{1}\left(\Pi_{0}\right)
$$

for some $\beta(\mu)>0$, see Section 3 . 
As for the structure of our paper, in Sections 2 and 3 we will prove the relationship

$$
\sigma_{\text {ess }}\left(A_{\bullet}\right)=\sigma_{\text {ess }}(A) \cup \sigma_{\text {di }}\left(A_{0}\right) \text {. }
$$

The proof of this formula consists of two steps. In Section 2 we construct a parametrix for the operator $A_{\bullet}-\lambda$ under the assumption $\lambda \notin \sigma_{\text {ess }}(A) \cup \sigma_{\text {di }}\left(A_{0}\right)$, and in Section 3 we use a quite standard construction of a Weyl sequence for the operator $A_{\bullet}$, when $\lambda \in \sigma_{\text {ess }}(A)$ or $\lambda \in \sigma_{\text {di }}\left(A_{0}\right)$.

Formula (1.15) shows that the non-compact perturbation $\Pi$ • of the domain $\Pi$ may add a family of isolated points to the essential spectrum on the intact domain. As verified in Theorem 3.2, these points are accumulation points of the point spectrum $\sigma_{\mathrm{po}}\left(A_{\bullet}\right)$, but unfortunately we are not at the moment able to show whether they are eigenvalues of infinite multiplicity or not.

If the outlet $\Pi$ is a straight cylinder and $\sigma_{\text {ess }}(A)=\left[\lambda_{\dagger},+\infty\right)$, then the new component of the spectrum consists of a finite number of eigenvalues belonging to $\sigma_{\mathrm{di}}\left(A_{0}\right) \subset\left(0, \lambda_{\dagger}\right)$, i.e. below the cut-off value $\lambda_{\dagger}$. For a periodic quasi-cylinder $\Pi$, the spectrum (1.6) may have gaps and $\sigma_{\mathrm{di}}\left(A_{0}\right)$ may contain points in these gaps. In Section 4 we will provide examples of both types of eigenvalues and also discuss possible generalizations of the problem setting.

We finish this section by a short discussion comparing our work with the literature on the Schrödinger equation. Non-periodic perturbations of the Schrödinger equation with periodic potentials have been investigated in many papers (see for example $[10,11,14,15]$ and others) especially in the case of the scattering problem in a system with "sparse bumps". In spite of some similarity of the geometric situation, our approach and results differ from these studies in many respects. In particular in our situation the essential spectrum of the unperturbed problem has band-gap structure and we are able to detect points of the essential spectrum of the perturbed problem, which are situated inside the gaps, not only below the essential spectrum as in the case of sparse bumps in the Schrödinger equation.

Our approach for finding the spectrum $\sigma_{\text {ess }}\left(A_{\bullet}\right)$ is standard, namely we will construct a singular Weyl sequence and a right parametrix in the regularity field. Because of the periodic geometry, these constructions need quite different arguments from those in $[14,15]$. In fact our techniques would apply to the Schrödinger equation having a potential with "stationary" behavior at infinity in the terminology of $[10]$; this is analogous with our fully periodic case. However, we consider only deterministic sparse potentials, contrary to the random ones in $[10,11]$.

\section{Constructing a parametrix.}

In this section we prove the relation

$$
\sigma_{\text {ess }}\left(A_{\bullet}\right) \subset \sigma_{\text {ess }}(A) \cup \sigma_{\text {di }}\left(A_{0}\right) .
$$

To this end, we assume

$$
\lambda \notin \sigma_{\mathrm{ess}}(A) \cup \sigma_{\mathrm{di}}\left(A_{0}\right)
$$

and construct a parametrix for the operator $A_{\bullet}-\lambda$ (cf. (1.8)) of the problem

$$
\begin{aligned}
-\Delta u_{\bullet}(x)-\lambda u_{\bullet}(x) & =f_{\bullet}(x), \quad x \in \Pi_{\bullet}, \\
u_{\bullet}(x) & =0, \quad x \in \partial \Pi_{\bullet},
\end{aligned}
$$

in other words a continuous operator

$$
R_{\bullet}(\lambda): L^{2}(\Pi) \rightarrow H^{2}\left(\Pi_{\bullet}\right) \cap H_{0}^{1}\left(\Pi_{\bullet}\right)
$$


such that the mapping

$$
\left(A_{\bullet}-\lambda\right) R_{\bullet}(\lambda)-1: L^{2}\left(\Pi_{\bullet}\right) \rightarrow L^{2}\left(\Pi_{\bullet}\right)
$$

is compact. This implies that the operator $A_{\bullet}-\lambda$ is Fredholm and therefore $\lambda \notin$ $\sigma_{\text {ess }}(A)$. Hence, by the Fredholm alternative, the problem (2.3) with the right-hand side $f_{\bullet} \in L^{2}\left(\Pi_{\bullet}\right)$ has a solution $u_{\bullet} \in H^{2}\left(\Pi_{\bullet}\right) \cap H_{0}^{1}\left(\Pi_{\bullet}\right)$, if and only if the compatibility conditions

$$
\left(f_{\bullet}, v_{\bullet}\right)_{\Pi_{\bullet}}=0 \quad \forall v_{\bullet} \in \operatorname{ker}\left(A_{\bullet}-\lambda\right)
$$

are satisfied. Here, the kernel, $\operatorname{ker}\left(A_{\bullet}-\lambda\right)$, is a finite dimensional space of solutions of the problem $(1.8)$ in $\left.H^{2}(\Pi)_{\bullet}\right) \cap H_{0}^{1}\left(\Pi_{\bullet}\right)$, due to the existence of the parametrix.

Since $\lambda \notin \sigma_{\text {ess }}(A)$, a parametrix $R(\lambda)$ of the operator $A-\lambda$ for the problem in $\Pi$ exists, see [16], $[21, \S 3.4, \S 5.1]$. We introduce the $C^{\infty}$-smooth cut-off functions $\chi: \mathbb{R} \rightarrow[0,1]$ and $X: \mathbb{R} \rightarrow[0,1]$,

$$
\begin{aligned}
& \chi(z)=1 \text { for } z \in(0,1) \text { and } \chi(z)=0 \text { for } z \notin\left(-\frac{1}{2}, \frac{3}{2}\right), \\
& X(z)=1-\sum_{j \in \mathbb{N}} \chi_{j}(z), \text { where } \chi_{j}(z)=\chi\left(z-L_{j}\right) .
\end{aligned}
$$

Setting $X(y, z):=X(z)$ for $(y, z) \in \Pi$, we have $f=X f_{\bullet} \in L^{2}(\Pi)$ and clearly also

$$
\left\|f ; L^{2}(\Pi)\right\| \leq\left\|f_{\bullet} ; L^{2}(\Pi)\right\| .
$$

Let $u_{b}=R(\lambda) f \in H^{2}(\Pi) \cap H_{0}^{1}(\Pi)$ and $u_{\sharp}=X u_{b} \in H^{2}\left(\Pi_{\bullet}\right) \cap H_{0}^{1}\left(\Pi_{\bullet}\right)$. We have

$$
\begin{aligned}
& -\Delta u_{\sharp}-\lambda u_{\sharp}-f_{\bullet} \\
= & -X(\Delta+\lambda) R(\lambda) X f_{\bullet}+X^{2} f_{\bullet}+\left(1-X^{2}\right) f_{\bullet}-[\Delta, X](\Delta+\lambda) R \lambda X f \bullet \\
= & -X((A-\lambda) R(\lambda)-1) X f_{\bullet}+\sum_{j \in \mathbb{N}} f_{j}, f_{j}=\chi_{j}^{2} f_{\bullet}-\left[\Delta, \chi_{j}\right] u_{b},
\end{aligned}
$$

where according to (2.6) the support of $f_{j}$ is contained in $\theta^{j}=\left\{x \in \overline{\Pi_{0}}:-\frac{1}{2} \leq\right.$ $\left.z-L_{j} \leq \frac{3}{2}\right\}$ and the estimate

$$
\sum_{j \in \mathbb{N}}\left\|f_{j} ; L^{2}\left(\theta^{j}\right)\right\|^{2} \leq c\left(\left\|f_{\bullet} ; L^{2}\left(\Pi_{\bullet}\right)\right\|^{2}+\left\|u_{b} ; H^{2}(\Pi)\right\|^{2}\right) \leq C\left\|f_{\bullet} ; L^{2}\left(\Pi_{\bullet}\right)\right\|^{2}
$$

holds true.

In order to compensate the terms $f_{j}$ in $(2.7)$ we need to derive more precise information on the problem

$$
\begin{aligned}
-\Delta u_{0}(x)-\lambda u_{0}(x) & =f_{0}(x), \quad x \in \Pi_{0}, \\
u_{0}(x) & =0, \quad x \in \partial \Pi_{0} .
\end{aligned}
$$

Because of the assumption (2.2), the operator of the problem (2.9),

$$
A_{0}-\lambda: H^{2}\left(\Pi_{0}\right) \cap H_{0}^{1}\left(\Pi_{0}\right) \rightarrow L^{2}\left(\Pi_{0}\right)
$$

is an isomorphism. We introduce the weighted spaces $L_{\beta}^{2}\left(\Pi_{0}\right)$ and $W_{\beta}^{q}\left(\Pi_{0}\right)$ as the completions of the linear set $C_{0}^{\infty}\left(\overline{\Pi_{0}}\right)$ with respect to the norms

$$
\begin{aligned}
& \left\|f_{0} ; L_{\beta}^{2}\left(\Pi_{0}\right)\right\|=\left\|e^{\beta|z|} f_{0} ; L^{2}\left(\Pi_{0}\right)\right\|, \\
& \left\|u_{0} ; W_{\beta}^{q}\left(\Pi_{0}\right)\right\|=\left(\sum_{p=0}^{q}\left\|\nabla^{p} u_{0} ; L^{2}\left(\Pi_{0}\right)\right\|^{2}\right)^{1 / 2},
\end{aligned}
$$


where $\beta \in \mathbb{R}$ and $q=1,2$ are the weight and smoothness indices and $\nabla^{p} u_{0}$ is the collection of all partial derivatives of $u_{0}$ of order $p$. Clearly, $L_{0}^{2}\left(\Pi_{0}\right)=L^{2}\left(\Pi_{0}\right)$ and $W_{0}^{q}\left(\Pi_{0}\right)=H^{q}\left(\Pi_{0}\right)$ in the case $\beta=0$, but for $\beta>0$, the spaces contain only functions with exponential decay at infinity. By $W_{\beta, 0}^{1}\left(\Pi_{0}\right)$ we denote the subspace of $W_{\beta}^{1}\left(\Pi_{0}\right)$ consisting of functions which vanish in $\partial \Pi_{0}$.

Obviously, the operator

$$
W_{\beta}^{2}\left(\Pi_{0}\right) \cap W_{\beta, 0}^{1}\left(\Pi_{0}\right) \ni u_{0} \mapsto \mathcal{O}_{\beta}(\lambda) u_{0}=: f_{0} \in L_{\beta}^{2}\left(\Pi_{0}\right)
$$

is continuous for all $\beta \in \mathbb{R}$. If additional conditions are posed on the index $\beta$, it follows from the results in $[16],[21, \S 3.4]$ that the operator $(2.11)$ can gain certain desirable properties: in particular, there exists a number $\beta(\lambda)>0$ such that if

$$
|\beta| \leq \beta(\lambda)
$$

then the operator (2.11) is an isomorphism (notice that in the case $\beta=0$, the mapping (2.11) coincides with the operator (2.10), which is an isomorphism due to our assumption on $\lambda)$.

The number $\beta(\lambda)$ can be found as follows. For a fixed $\lambda$, problem (1.9) gives rise to a polynomial (quadratic) operator pencil $\eta \mapsto \mathfrak{A}_{\lambda}(\eta)$, cf. [7, Ch. 1], [21, §1.2]. The condition $\lambda \notin \sigma_{\text {ess }}(A)$ in (2.2) implies that there is no $\eta$-spectrum of $\mathfrak{A}_{\lambda}$ in the segment $[-\pi, \pi] \subset \mathbb{R} \subset \mathbb{C}$. Due to the analytic Fredholm alternative, see e.g. $[7$, Thm. 1.5.1], the $\eta$-spectrum of $\mathfrak{A}_{\lambda}$ consists of normal eigenvalues and has no finite accumulation points. Furthermore, the $\eta$-spectrum is evidently $2 \pi$-periodic with respect to the real variable. Thus, there exists $\beta(\lambda)>0$ such that the rectangle $\{\eta \in \mathbb{C}:|\operatorname{Re} \eta| \leq \pi,|\operatorname{Im} \eta| \leq \beta(\lambda)\}$ does not contain points of the $\eta$-spectrum of $\mathfrak{A}_{\lambda}$. By [16, Thms. 4-6], $[21, \S 3.4, \S 5.1]$, the operators $(2.11)$ with $\beta \in(-\beta(\lambda), \beta(\lambda))$ have the same properties and therefore inherit the isomorphism property of $(2.10)$.

Finally, we apply the above solvability result in weighted spaces to the construction of the parametrix. This is based on the fact that the supports of the functions in $(2.7)$,

$$
\Pi_{0} \ni x \mapsto f_{j, 0}(y, z)=f_{j}\left(y, z+L_{j}\right)
$$

are compact, and they thus belong to $L_{\beta}^{2}\left(\Pi_{0}\right)$ for any $\beta$, and consequently, the problem (2.9) with $f_{0}=f_{j, 0}$ has a unique solution $u_{j, 0} \in W_{\beta(\lambda)}^{2}\left(\Pi_{0}\right) \cap W_{\beta(\lambda), 0}^{2}\left(\Pi_{0}\right)$ decaying exponentially. We define for every $j \in \mathbb{N}$ the cut-off function $X_{j}: \mathbb{R} \rightarrow[0,1]$ by

$$
X_{j}(z)=\mathcal{X}\left(z-L_{j-1}-1\right)\left(1-\mathcal{X}\left(z-L_{j-1}+1\right)\right)
$$

where

$$
\mathcal{X}(z)=0 \text { for } z<0 \text { and } \mathcal{X}(z)=1 \text { for } z>1
$$

so that

$$
\begin{aligned}
& X_{j}(z)=1 \text { for } z \in\left(L_{j-1}+2, L_{j}-1\right) \text { and } \\
& X_{j}(z)=0 \text { for } z \notin\left(L_{j-1}+1, L_{j}\right) .
\end{aligned}
$$

Let

$$
u_{\bullet}=R_{\bullet}(\lambda) f_{\bullet}=u_{\sharp}+\sum_{j \in \mathbb{N}} X_{j} u_{j},
$$


where $u_{j}(y, z)=u_{j, 0}\left(y, z-L_{j}\right)$ and $X_{j}(y, z):=X_{j}(z)$. The identities (2.6), (2.7) and (2.15) yield

$$
-\Delta u_{\bullet}-\lambda u_{\bullet}-f_{\bullet}=X((A-\lambda) R(\lambda)-1) X f_{\bullet}-\sum_{j \in \mathbb{N}}\left[\Delta, X_{j}\right] u_{j} .
$$

The operator $X((A-\lambda) R(\lambda)-1) X$ is compact in $L^{2}\left(\Pi_{\bullet}\right)$, since $R(\lambda)$ is a parametrix. We denote the last sum in (2.16) by $K f_{\bullet}$ and verify that the operator $K: L^{2}\left(\Pi_{\bullet}\right) \rightarrow$ $L^{2}\left(\Pi_{\bullet}\right)$ is compact, too.

Due to $(2.8),(2.14)$ and the inclusion $u_{j, 0} \in W_{\beta(\lambda)}^{2}\left(\Pi_{0}\right)$ we have

$$
\begin{aligned}
& \sum_{j \in \mathbb{N}}\left(e^{2 \beta(\lambda) \ell_{j}}\left\|\left[\Delta, X_{j}\right] u_{j} ; H^{1}\left(\varpi\left(L_{j-1}+1\right)\right)\right\|^{2}\right. \\
+ & \left.e^{2 \beta(\lambda) \ell_{j+1}}\left\|\left[\Delta, X_{j}\right] u_{j} ; H^{1}\left(\varpi\left(L_{j+1}-1\right)\right)\right\|^{2}\right) \\
\leq & c \sum_{j \in \mathbb{N}} \| u_{j, 0} ; W_{\beta(\lambda)}^{2}\left(\varpi\left(L_{j-1}+1-L_{j}\right) \cup\left(\varpi\left(L_{j+1}-1-L_{j}\right)\right) \|^{2}\right. \\
\leq & c \sum_{j \in \mathbb{N}}\left\|u_{j, 0} ; W_{\beta(\lambda)}^{2}\left(\Pi_{0}\right)\right\|^{2} \\
\leq & c \sum_{j \in \mathbb{N}}\left\|f_{j, 0} ; L_{\beta(\lambda)}^{2}\left(\Pi_{0}\right)\right\|^{2} \leq C\left\|f_{\bullet} ; L^{2}\left(\Pi_{\bullet}\right)\right\|^{2}
\end{aligned}
$$

Let $\Theta=\cup_{j \in \mathbb{Z}} \cup_{ \pm} \varpi\left(L_{j} \pm 1\right)$ be the union of the cells appearing on the left of (2.17). We define the space $\mathcal{H}_{\alpha}(\Theta)$ as the space of functions on $\Theta$ with weighted norm

$$
\left\|g ; \mathcal{H}_{\alpha}(\Theta)\right\|=\left(\sum_{j \in \mathbb{N}} \alpha_{j}^{2}\left\|g ; H^{1}\left(\cup_{ \pm} \varpi\left(L_{j} \pm 1\right)\right)\right\|^{2}\right)^{1 / 2}
$$

where we can choose the sequence $\left\{\alpha_{j}\right\}_{j \in \mathbb{N}}$ tending to $+\infty$ for example as

$$
\alpha_{j}=e^{\beta(\lambda) \ell_{j}} .
$$

The embedding $\mathcal{H}_{\alpha}(\Theta) \hookrightarrow L^{2}(\Theta)$ is compact, because it can be written as a sum of a compact operator (considering the restriction of the functions of $\mathcal{H}_{\alpha}(\Theta)$ to finitely many domain components $\varpi\left(L_{j} \pm 1\right)$ ) and another one with operator norm, which can be made as small as one wishes (due to the coefficients $\alpha_{j}$ in the remaining components).

According to (2.17), the operator $K$ is compact as well. Consequently, the operator $(A-\lambda) R_{\bullet}(\lambda)-1$ from the left of $(2.16)$ is also compact.

We formulate the proven fact as the following result.

Theorem 2.1. Assume that (2.2) holds for the spectral parameter $\lambda$. Then, the operator $A_{\text {. }}$ of the problem (1.8) has a parametrix determined by the formula (2.15).

\section{Constructing a Weyl sequence.}

We now complete the proof of the formula (1.15) by verifying the relation inverse to (2.1). For any $\lambda \in \sigma_{\text {ess }}(A)$, the problem (1.4) in the intact quasicylinder $\Pi$ has a bounded solution, the Floquet wave $u(x)=e^{i \eta z} U(y, z)$, where $U$ is the eigenfunction 
of the problem (1.9) corresponding to $\Lambda(\eta)=\lambda$ (cf. (1.6)). We compose a Weyl sequence by using translations of the functions

$$
v^{n}(y, z)=\left\|\mathcal{X}_{n} u ; L^{2}(\Pi)\right\|^{-1} \mathcal{X}_{n}(z) u(y, z),
$$

where $\mathcal{X}_{n}$ is the cut-off-function (see (2.13))

$$
\mathcal{X}_{n}(z)=\mathcal{X}(n-|z|) .
$$

Notice that by definition we have

$$
\begin{aligned}
& \left\|\mathcal{X}_{n} u ; L^{2}(\Pi)\right\|^{2} \geq 2(n-1)\left\|U ; L^{2}(\varpi)\right\|^{2} \\
& \left\|(A-\lambda) \mathcal{X}_{n} u ; L^{2}(\Pi)\right\|^{2}=\left\|\left[\Delta, \mathcal{X}_{n}\right] u ; L^{2}(\Pi)\right\|^{2} \leq c,
\end{aligned}
$$

because the commutator $\left[\Delta, \mathcal{X}_{n}\right]$ is supported in the union of $\overline{\varpi(n)}$ and $\overline{\varpi(1-n)}$.

Since $\ell_{j} \rightarrow+\infty$ as $j \rightarrow+\infty$, we find a monotonely increasing sequence $\left\{j^{n}\right\} \subset \mathbb{N}$ such that $\ell_{j^{n}} \geq 2 n+1$. Owing to (3.1)-(3.3), the functions

$$
\text { П. } \ni(y, z) \mapsto v^{n}\left(y, z-L_{j}+n\right)
$$

are well-defined and form a singular Weyl sequence for the operator $A_{\bullet}$ at the point $\lambda$, which thus falls into $\sigma_{\text {ess }}\left(A_{\bullet}\right)$, see [4, Thm. 9.1.2], [24, Thm.VII.12].

Let now $\lambda \in \sigma_{\mathrm{di}}\left(A_{0}\right)$ and let $w \in H^{2}\left(\Pi_{0}\right) \cap H_{0}^{1}\left(\Pi_{0}\right)$ be the corresponding eigenfunction of the problem (1.12), normalized in $L^{2}\left(\Pi_{0}\right)$. Changing $u$ into $w$ in (3.1) yields a singular Weyl sequence and thus the inclusion $\lambda \in \sigma_{\text {ess }}\left(A_{\bullet}\right)$ follows as above. We have thus proven the converse of (2.1), and hence we can formulate the main result of our paper:

Theorem 3.1. The formula (1.15) is valid, that is, $\sigma_{\mathrm{ess}}\left(A_{\bullet}\right)=\sigma_{\mathrm{ess}}(A) \cup \sigma_{\mathrm{di}}\left(A_{0}\right)$.

We complete this section by deriving some more accurate information on the spectrum.

Given $\mu \in \sigma_{\mathrm{di}}\left(A_{0}\right)$, the exponential decay in (1.14) yields the inequalities

$$
\begin{aligned}
& \left|\left\|\mathcal{X}_{n} w ; L^{2}\left(\Pi_{0}\right)\right\|^{2}-1\right| \leq c e^{-\beta(\mu) n} \\
& \left\|\left(A_{0}-\lambda\right) \mathcal{X}_{n} w ; L^{2}\left(\Pi_{0}\right)\right\|^{2} \leq c e^{-2 \beta(\mu) n},
\end{aligned}
$$

so that $\lambda \in \sigma_{\mathrm{di}}\left(A_{\bullet}\right)$ is seen to be an accumulation point of the point spectrum $\sigma_{\mathrm{po}}\left(A_{\bullet}\right)$ of the problem (1.3).

Theorem 3.2. Let $\mu \in \sigma_{\mathrm{di}}\left(A_{0}\right)$. Given arbitrary $N \in \mathbb{N}$ and $\varepsilon>0$, the total multiplicity of the point spectrum of $A$. contained in the interval

$$
v^{\varepsilon}(\lambda)=[\mu-\varepsilon, \mu+\varepsilon]
$$

is at least $N$.

Proof. Let $E_{\bullet}(t) d t$ be the spectral measure generated by $A_{\bullet}$, see [4, Ch.6], [24, Sect. VII.2]. If $\varepsilon$ is small and $v^{\varepsilon}(\mu) \cap \sigma_{\text {ess }}(A)=\varnothing$, then this is a discrete measure on $v^{\varepsilon}(\mu)$. Thus, we have to verify that

$$
d_{\bullet}^{\varepsilon}(\lambda):=\operatorname{dim}\left(P_{\bullet}^{\varepsilon}(\lambda) \mathcal{D}\left(A_{\bullet}\right)\right) \geq N,
$$

where $P_{\bullet}^{\varepsilon}(\lambda)$ is the orthogonal projection

$$
P_{\bullet}^{\varepsilon}(\lambda)=\int_{v^{\varepsilon}(\lambda)} E_{\bullet}(t) d t .
$$


Since $\ell_{j} \rightarrow+\infty$ as $j \rightarrow+\infty$, we can select a monotonely increasing sequence $\left\{j^{n}\right\} \in \mathbb{N}$ such that $\ell_{j^{n}-1}, \ell_{j^{n}} \geq n+1$ and thus the functions

$$
w^{n}(y, z)=\mathcal{X}_{n}\left(z-L_{j^{n}}\right) w\left(y, z-L_{j^{n}}\right)
$$

belong to $\mathcal{D}\left(A_{\bullet}\right)$. Moreover, the functions

$$
v^{n}=\left\|w^{n} ; L^{2}\left(\Pi_{0}\right)\right\|^{-1} w^{n}, \quad n \in \mathbb{N},
$$

are orthonormalized in $L^{2}\left(\Pi_{\bullet}\right)$ because $\operatorname{supp} v^{n} \cap \operatorname{supp} v^{m}=\varnothing$ for $n \neq m$. Then,

$$
\begin{aligned}
& \delta_{n, m}-\left(P_{\bullet}^{\varepsilon}(\lambda) v^{n}, P_{\bullet}^{\varepsilon}(\lambda) v^{m}\right)_{\Pi_{\bullet}}=\left(\left(1-P_{\bullet}^{\varepsilon}(\lambda)\right) v^{n}, v^{m}\right)_{\Pi_{\bullet}} \\
= & \frac{1}{4}\left(\left\|\left(1-P_{\bullet}^{\varepsilon}(\lambda)\right)\left(v^{n}+v^{m}\right) ; L^{2}\left(\Pi_{\bullet}\right)\right\|^{2}-\left\|\left(1-P_{\bullet}^{\varepsilon}(\lambda)\right)\left(v^{n}-v^{m}\right) ; L^{2}\left(\Pi_{\bullet}\right)\right\|^{2}\right) \\
(3.8)= & \frac{1}{4} \int_{\mathbb{R} \backslash v^{\varepsilon}(\lambda)} d \mu_{v^{n}+v^{m}}-\frac{1}{4} \int_{\mathbb{R} \backslash v^{\varepsilon}(\lambda)} d \mu_{v^{n}-v^{m}},
\end{aligned}
$$

where $\delta_{n, m}$ is the Kronecker symbol and $d \mu_{W}=\left(E_{\bullet} W, W\right)_{\Pi \bullet}$ is the non-negative scalar measure generated by $W \in \mathcal{D}\left(A_{\bullet}\right)$. Using relations $(3.5)$ and

$$
\begin{aligned}
& \int_{\mathbb{R} \backslash v^{\varepsilon}(\lambda)} d \mu_{v^{n} \pm v^{m}} \leq \frac{1}{\varepsilon^{2}} \int_{\mathbb{R} \backslash v^{\varepsilon}(\lambda)}(t-\lambda)^{2} d \mu_{v^{n}+v^{m}}(t) \\
\leq & \varepsilon^{-2}\left\|\left(A_{\bullet}-\lambda\right)\left(v^{n} \pm v^{m}\right) ; L^{2}\left(\Pi_{\bullet}\right)\right\|^{2}
\end{aligned}
$$

yields the bound $c \varepsilon^{-2} e^{-2 \beta(\lambda) \min \{m, n\}}$ for the moduli of the two integrals in (3.8). Thus, for fixed $\varepsilon>0, N \in \mathbb{N}$ and arbitrary $\delta>0$, we find $M$ such that

$$
\left|\left(P_{\bullet}^{\varepsilon}(\lambda) v^{n}, P_{\bullet}^{\varepsilon}(\lambda) v^{m}\right)_{\Pi \bullet}-\delta_{n, m}\right| \leq \delta
$$

for all $M \leq n, m<M+N$. In other words, for sufficiently small $\delta>0$, the functions $P_{\bullet}^{\varepsilon}(\lambda) v^{M}, \ldots P_{\bullet}^{\varepsilon}(\lambda) v^{M+N-1}$ are "almost orthonormal" and they in particular form a linearly independent sequence. This can happen, if and only if (3.7) is true.

Unfortunately, the authors do not know if $\lambda \in \sigma_{\text {ess }}\left(A_{0}\right)$ can be an eigenvalue with infinite multiplicity for the problem (1.8), although this possibility seems improbable.

\section{ExAmples AND Generalizations.}

Let us first demonstrate by some examples that the essential spectra $\sigma_{\text {ess }}\left(A_{\bullet}\right)$ and $\sigma_{\text {ess }}(A)$ of Theorem 3.1 indeed may be different. We start by discussing the possibility of having an eigenvalue $\lambda^{-} \in \sigma_{\mathrm{di}}\left(A_{0}\right)$, see (1.12), below the cut-off (minimum) $\lambda_{\dagger}$ of the essential spectrum $\sigma_{\text {ess }}(A)$. Our smoothness assumptions on the boundary

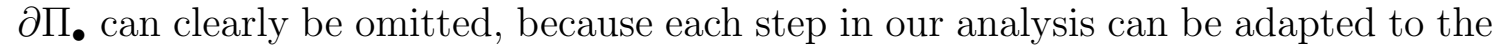
variational problem (1.5) and the one corresponding to (1.12):

$$
(\nabla w, \nabla v)_{\Pi_{0}}=\lambda(w, v)_{\Pi_{0}} \quad \forall v \in H_{0}^{1}\left(\Pi_{0}\right) .
$$

If $\Pi \subsetneq \Pi_{0}$ holds, we can apply the comparison principle, [12], which is a consequence of the max-min-principle [4, Thm. 10.2.2], [24, Sec. XIII.1], and which implies that enlarging the waveguide $\Pi_{0}$ yields at least one eigenvalue $\lambda^{-}$for $A_{0}$ in the interval $\left(0, \lambda_{\dagger}\right)$ below the essential spectrum. In view of Theorem 3.1, these remarks imply that $\lambda^{-} \in \sigma_{\text {ess }}\left(A_{\bullet}\right)$ although $\lambda^{-}<\lambda_{\dagger}$. 


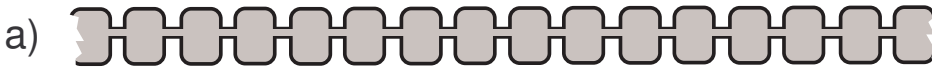 \\ b) $500000000000000 \mathrm{C}$}

FiguRE 4.1. Different geometries of the waveguide $\Pi$ yielding spectral gaps.

However, if $\Pi_{0} \subset \Pi$ and $\Pi=\omega \times(0,+\infty)$ is a cylindrical outlet, the discrete spectrum $\sigma_{\mathrm{di}}\left(A_{0}\right)$ is empty and, therefore, by Theorem 3.1,

$$
\sigma_{\mathrm{ess}}\left(A_{\bullet}\right)=\sigma_{\mathrm{ess}}(A)
$$

Next, we consider possible eigenvalues inside spectral gaps. We assume that $\Pi$ is a quasicylinder such that there exists a spectral gap $\gamma(n) \neq \varnothing$ between the bands $\beta(n)$ and $\beta(n+1),(1.13)$, and that the edges $\beta^{+}(n)$ and $\beta^{-}(n+1)$ are non-degenerate, i.e., the second derivatives satisfy $\partial_{\eta}^{2} \Lambda_{n}\left(\eta_{\max }\right)<0$ and $\partial_{\eta}^{2} \Lambda_{n+1}\left(\eta_{\min }\right)>0$. Then, according to [19], slightly diminishing the waveguide $\Pi_{0}$ in the case $\Pi_{0} \subsetneq \Pi$ (or enlarging it, if $\left.\Pi_{0} \supsetneq \Pi\right)$, gives rise to an eigenvalue inside the gap $\gamma(n)$ near its lower edge $\beta^{+}(n)$ (respectively, upper edge $\beta^{-}(n+1)$ ).

We complete the above argument by the remark that there are several known ways to open spectral gaps in periodic waveguides, based on controlling the shape of the periodicity cell:

$1^{\circ}$. Identical beads connected by a thin needle, Fig. 4.1,a). In this case the spectrum (1.6) consists of short bands separated by wide gaps as in Fig. 4.2,a); see [23].

$2^{\circ}$. Periodic perturbation by small voids of the cylindrical surface $\partial \Pi=\partial \omega \times \mathbb{R}$, see Fig. 4.1, b). Under certain conditions on the perturbation profile one can open short spectral gaps as in Fig. 4.2,b); see [17, 5, 18].

Notice that in both cases the band edges are non-degenerate.

Finally, if the Dirichlet boundary conditions are replaced by Neumann conditions, we have $\sigma_{\text {ess }}=[0,+\infty)$ for the Laplace operator in a straight cylinder, and formula (1.15) holds trivially true. However, in the case the domain is a quasicylinder, spectral gaps may appear, and our proofs of Theorems 2.1, 3.1 and 3.2 can easily be adapted to these and other boundary conditions. In particular, spectral gaps can be opened by the methods $1^{\circ}$ and $2^{\circ}$, cf. [17], [18] and [2].

$3^{\circ}$. Contrasting coefficients of the differential operators, see [8], [9], [26] and [1].

We remark that the methods $1^{\circ}$ and $3^{\circ}$ can directly be generalized to elliptic systems, for example the elasticity problem, see $[22,3,6]$, but there are serious obstacles to apply the approach $2^{\circ}$ in this way, and this has not been done in the literature yet.

We finish the paper by the remark that the results and their proofs directly generalize to domains in $\mathbb{R}^{d}$ having one or finitely many outlets to infinity: for example, a waveguide $\Omega$ could be composed of the semi-infinite outlet

$$
\Pi^{+}=\{x \in \Pi: z \geq 0\},
$$

where $\Pi$ is as before, and the attached bounded domain $\omega$ contained in the halfspace $\mathbb{R}_{-}^{d}=\{x: z<0\}$. A perturbed domain $\Omega$. could be defined in the same 
a)

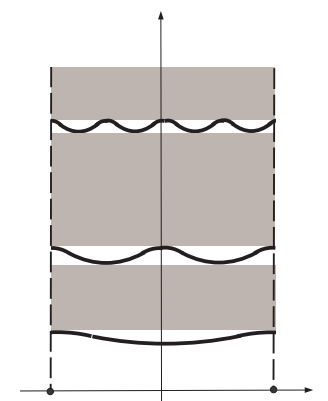

b)

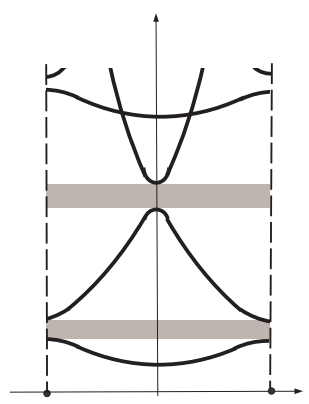

FigurE 4.2. Wide (a) and short (b) gaps of the essential spectrum (shaded).

way as around (1.7), and the results in Theorem 3.1 and 3.2 would hold as such, for equations and proofs where $\Pi$ is replaced by $\Omega$ and $\Pi_{\bullet}$ with $\Omega_{\bullet}$.

\section{REFERENCES}

[1] F.L. Bakharev, G.Cardone, S.A.Nazarov, J.Taskinen, Effects of Rayleigh Waves on the Essential Spectrum in Perturbed Doubly Periodic Elliptic Problems, Int. Eq. Oper. Th. 88 (2017), 373-386.

[2] F.L. Bakharev, S.A. Nazarov, K. Ruotsalainen, A gap in the spectrum of the NeumannLaplacian on a periodic waveguide, Applicable Analysis. (9) 88 (2012), 1889-1995.

[3] F.L. Bakharev, J.Taskinen, Bands in the spectrum of a periodic elastic waveguide, Zeitschrift Angew. Math. Phys. (2017), to appear.

[4] M.S. Birman, M.Z. Solomyak, Spectral theory of selfadjoint operators in Hilbert space. Math. Appl. (Soviet Ser.), D. Reidel Publishing Co., Dordrecht 1987.

[5] D.I. Borisov, K.V. Pankrashkin, Gap opening and splitting of the zone edges for waveguides coupled by a periodic system of small windows, Mat. Zametki (5) 93 (2013), 665-683. (English transl. Math. Notes (5-6) 93 (2013), 660-675.)

[6] G.Cardone, S.A.Nazarov, J.Taskinen, Spectra of open waveguides in periodic media. J. Functional Anal. 269 (2015), 2328-2364.

[7] I.C. Gohberg, M.G. Krein, Introduction to the Theory of Linear Nonself- Adjoint Operators in Hilbert Space. Nauka, Moscow 1965. (English transl. Amer. Math. Soc., Providence, RI 1969.)

[8] R. Hempel, K. Lienau, Spectral properties of periodic media in the large coupling limit, Communications in Partial Differential Equations (7-8) 25 (2000), 1445-1470.

[9] R. Hempel, O. Post, Spectral Gaps for Periodic Elliptic Operators with High Contrast: An Overview. Progress in Analysis, Proceedings of the 3rd International ISAAC Congress, Berlin $2001,577-587$.

[10] D. Hundertmark, W. Kirsch, Spectral theory of sparse potentials. Stochastic processes, physics and geometry: new interplays, I (Leipzig, 1999), 213-238, CMS Conf. Proc., 28, Amer. Math. Soc., Providence, RI, 2000

[11] V. Jakšić, P. Poulin, Scattering from Sparse Potentials: a Deterministic Approach, Analysis and Mathematical Physics, Trends in Mathematics 205-210.

[12] D.S. Jones, The eigenvalues of $\nabla^{2} u+\lambda u=0$ when the boundary conditions are given on semi-infinite domains. Proc. Camb. Phil. Soc. 49 (1953), 668-684.

[13] P. Kuchment, Floquet theory for partial differential equations. Oper. Theory Adv. Appl. 60, Birkhäuser, Basel 1993.

[14] S. Molchanov, B. Vainberg, Scattering on the system of sparse bumps: multidimensional case, Applicable Analysis 71 (1-4), 167-185.

[15] S. Molchanov, B. Vainberg, Spectrum of multidimensional Schrödinger operators with sparse potentials, Analytical and Computational Methods in Scattering and Applied Mathematics (Newark, DE, 1998), 231-254, Chapman \& Hall/CRC. 
[16] S.A. Nazarov, Elliptic boundary value problems with periodic coefficients in a cylinder. Izv. Akad. Nauk SSSR. Ser. Mat. (1) 45 (1981), 101-112. English transl. Math. USSR. Izvestija (1) 18 (1982), 89-98.

[17] S.A. Nazarov, Opening a gap in the continuous spectrum of a periodically perturbed waveguide. Mat. Zametki. (5) 87 (2010), 764-786. (English transl.: Math. Notes (5) 87 (2010), $738-756)$.

[18] S.A. Nazarov, Asymptotic behavior of spectral gaps in a regularly perturbed periodic waveguide. Vestnik St.-Petersburg Univ. (2) 7 (2013), 54-63. (English transl.: Vestnik St.-Petersburg Univ. Math. (2) 46 (2013), 89-97.)

[19] S.A. Nazarov, Asymptotics of eigenvalues in spectral gaps under regular perturbations of walls of a periodic waveguide, J. Math. Sci (4) 226 (2017), 402-444.

[20] S.A. Nazarov, Open waveguides in a thin Dirichlet lattice: I.Asymptotic structure of the spectrum. Zh. Vychisl. Mat. i Mat. Fiz. (1) 57 (2017), 127-146. (English transl.: Comput. Math. and Math. Physics (1) 57 (2017), 156-174.)

[21] S.A. Nazarov, B.A. Plamenevsky, Elliptic problems in domains with piecewise smooth boundaries. Walter de Gruyter, Berlin, New York, 1994.

[22] S.A.Nazarov, K.Ruotsalainen, J.Taskinen, Essential spectrum of a periodic elastic waveguide may contain arbitrarily many gaps Applicable Anal. (1) 89 (2010), 109-124.

[23] O. Post, Periodic manifolds with spectral gaps. J. Diff. Eq 187 (2003), 23-45.

[24] M. Reed, B. Simon, Methods of modern mathematical physics, Vol. I, IV. Academic Press 1980, 1978

[25] M.M. Skriganov, Geometric and arithmetic methods in the spectral theory of multidimensional periodic operators. Tr. Math. Inst. Steklov., 171 (1985), 3-122. (English transl. Proc. Steklov Inst. Math. 171 (1987), 1-121.)

[26] V.V. Zhikov, On gaps in the spectrum of some elliptic operators in divergent form with periodic coefficients. St. Petersbg. Math. J. (5) 16 (2005), 773-790;

Saint-Petersburg State University, Universitetskaya nab., 7-9, St. Petersburg, 199034, Russia, AND

Institute of Problems of Mechanical Engineering RAS, V.O., Bolshoj Pr., 61, St. Petersburg, 199178, Russia

E-mail address: s.nazarov@spbu.ru, srgnazarov@yahoo.co.uk

Department of Mathematics and Statistics, P.O.Box 68, University of Helsinki, 00014 Helsinki, Finland

E-mail address: jari.taskinen@helsinki.fi 